\title{
Linking data and models
}

\author{
GeRRIt LoHMANN
}

Alfred Wegener Institute for Polar and Marine Research, Bremerhaven, Germany; gerrit.lohmann@awi.de

Paleo-data and models have the potential for a truly symbiotic relationship. One application of paleoclimate data is to validate state-of-the-art coupled climate models for past time slices and specific climate transitions. Analyzing proxy-reconstructed paleoclimate records and models in tandem allows for the evaluation of climate transitions through the analysis of forcing and feedback mechanisms in past and future climate changes. In return, model simulations can aid in the interpretation of the causes of observed variations in paleoclimate data. Climate simulations enable a separation of the externally forced climate signal from internal variability (to the extent that the signal is distinguishable from the noise), something that cannot be achieved using proxy data alone. To become effective, these mechanisms require that data and model simulations can be compared in a meaningful way.

This special section of PAGES News highlights the importance of validating the results of individual reconstructions and simulations, and showcases a variety of methods that can be used for their comparison.

\section{Spatial and temporal obstacles}

Comparisons between paleoclimate and model data are hindered by the different characteristics of each data set. Model output is less reliable at small spatial scales, while some proxy data can be representative of only single sites. An important task is to develop methodologies for coping with these characteristics. This will allow subsequent, unbiased comparisons between simulations and proxy data that explicitly take into account the estimated errors in the proxy reconstructions and the small-scale resolution issues with models.

In general, proxy data are sampled at discrete spots in the areal dimensions of the Earth's surface and record the temporal dimension well. The vertical component of the environmental signal is only marginally captured, for example, by near-surface, thermocline, and nearbottom living marine organisms, or by sample transects across topographic gradients. While the reconstructions usually contain many points in time, the data will only be available at a limited number of discrete spatial locations. On the other hand, the spatial scale of recent global cli- mate models (less than $300 \mathrm{~km}$ ) enables the combined spatio-temporal domain to be explored.

\section{Model-data advances}

The comparison of paleoclimate and model data can be carried out in a number of ways. The simplest approach is to subsample the model output fields, picking out data only from those locations and seasons for which paleo-reconstructions exist for comparison. However, before a valid comparison can be made, it must be confirmed that the climate response is being compared on similar spatial and temporal scales.

There are two key statistical methods that synchronize the spatial scale of the model simulation and proxy reconstruction. The upscaling technique identifies the underlying large-scale processes, e.g., the teleconnections that control lowfrequency variations observed in many proxy records. In this case, the statistical method brings the climate modes and shifts, such as those observed in the past 100 years, into a long-term context. The upscaling method can even be used to reconstruct synoptic conditions related to reconstructed ice core data (Rimbu et al., p. 5). The large-scale signal, which can be remote from the local proxy record, can then be compared with model simulations. In a similar direction, a variational approach can be used to connect the different scales of local paleo-information together with a dynamically consistent spatial smoothing (Kühl et al., p. 8). This is in contrast to the downscaling technique, where large-scale information obtained by a model simulation can be "zoomed in" to the smaller scale of proxy climate information (Raible et al., p. 10; Meyer and Wagner, p. 12).

Comparison of model simulations and data reconstructions can additionally aid in clarifying the true environmental signals recorded by proxies. It has been proposed that proxy parameters be included as tracers in models and create numerical simulations of proxy generation and burial in all available archives (i.e., synthetic marine, terrestrial and ice core), to compare them with real archives. This idea developed from the need for a mechanistic understanding of how environmental conditions are transferred to the archive. Proxy parameters that are found in a number of different archives, e.g., ${ }^{18} \mathrm{O}$ in foraminifera and ice cores, are particularly useful in this approach, as they provide information on how the climate signal is recorded within the one proxy in different climate components (ocean, ice). An example of modeling of marine $\Delta^{14} \mathrm{C}$, and subsequent comparison with marine reservoir age, is presented by Butzin et al. (p. 13). The idea of generating pseudo-proxy records is also an important component of the new Paleo-Reconstruction Challenge (see Ammann, PAGES News 2008, 16(1): 4).

A variety of comparison techniques allow many additional objectives to be achieved through comparison of model simulations and proxy reconstructions. Identifying key regions where specific climate signals are likely to be recorded can optimize sampling efforts for future proxy reconstructions. It would be useful, for example, to explore targeted climate hot spots through a combined use of paleoclimate reconstructions and model-simulated climate data. Signal-to-noise ratios, or inverse methods, may be used to evaluate locations where climate phenomena can best be detected.

Comparison can also be made between different climate simulations, in order to assess whether additional forcings raise the levels of variability to a similar extent in both the simulated and reconstructed climates (Goosse et al., p. 15). Model results may also provide information on the onset, duration and magnitude of climate events, similar to how they may be expressed in proxy-based reconstructions (Wiersma et al., p. 16). Another approach is to test the robustness of model results through comparison of multiple model outputs with data (Otto-Bliesner and Brady, p. 18). Data-model comparisons can also enable the estimation of climate sensitivity (Schneider von Deimling et al., p. 20), which is important for predictions of future temperature rise based on current $\mathrm{CO}_{2}$ projections.

Climate sensitivity estimates are reliant on estimates of past climate forcings. Data-model comparison can additionally be useful in reducing uncertainties in these past forcings (Crowley et al., p. 22). A slightly different approach is to run the model using the proxy data and to then use the model to obtain a dynamically consistent interpretation of the data. This approach has been used, for example, to clarify the temperature and ocean circulation regime during the Pliocene (Chandler 
et al., p. 24). Models can also be tested against past and present scenarios (van Oldenborgh, p. 26; Mudelsee and Girardin, p. 28), which again helps to improve climate predictability.

\section{Format and availability of data}

For a data-model comparison, the proxyreconstruction data needs first to be combined into one large data set with wide temporal and spatial coverage. This requires detailed information on sampling methods, age models and representativeness of proxy data. Fundamental to the continuation of the model-data comparison effort is the availability of and accessibility to data sets of proxy reconstructions and model output (Dittert et al., p. 30). Many recent data-modeling efforts have utilized proxy data sets made available in data archives.

\section{Scientific education-bridging the gaps between disciplines}

Earth System science is traditionally split into various disciplines and sub-disciplines. Overall, the diversity of expertise provides a solid base for interdisciplinary research. However, to gain holistic insights into the Earth System requires the integration of observations, paleoclimate data and climate modeling. These different approaches of Earth System science are rooted in the different disciplines (geology, physics, meteorology, oceanography, etc.), which cut across a broad range of timescales. It is therefore necessary to link these disciplines at a relatively early stage in $\mathrm{MSc} / \mathrm{PhD}$ programs. The linking of data and modeling would enable graduate students from a variety of disciplines to cooperate and exchange views on the common theme of Earth System science, and lead to a better understanding of local processes within a global context.

Computational and conceptual models of the Earth System provide the ability to investigate different scenarios in biogeochemistry, such as the carbon cycle, the structure of marine sediments, and isotope distribution in climate components. Statistical analysis further provides a synthesis, comparison and interpretation of paleoclimate and simulated data. Training and education, particularly in time-series analysis, data exploration, process understanding and model interpretation, should all be key components of future education.

\section{Northern hemisphere atmospheric blocking in ice core accumulation records from northern Greenland}

Norel Rimbu, G. Lohmann and K. Grosfeld

Alfred Wegener Institute for Polar and Marine Research, Bremerhaven, Germany; Norel.Rimbu@awi.de

Atmospheric blocking is a large-scale, midlatitude atmospheric phenomenon often associated with persistent quasi-stationary, synoptic-scale, high-pressure systems. The formation, maintenance and collapse of atmospheric blocking cause large-scale circulation anomalies and strongly impact weather patterns. Therefore, blocking regimes constitute a significant climatological feature.

Northern hemisphere blocking shows important variability at different timescales. Blocking frequencies have shown a downward (upward) trend over Atlantic and European (west Pacific) sectors. Su- perimposed on these linear trends, blocking frequencies show significant interannual and decadal variation (Barriopedro et al., 2006). However, in these studies, the blocking variability was derived over the relatively short time period covered by observational data with daily resolution. Here, we present the first attempt to directly relate interannual and decadal variability of several high-resolution snow accumulation records from northern Greenland with northern hemisphere atmospheric blocking. First, we investigate the relationship between atmospheric blocking and accumulation variability dur- ing the period covered by both accumulation and high-resolution (daily) observational data. Based on this relationship, we then discuss the blocking variability over the last $400 \mathrm{yr}$ based only on the accumulation variability.

As an example of blocking circulation, we present in Figure 1a the 500-hPa geopotential height (shaded) and horizontal wind (vectors) during the mature phase (3 February 1975) of the blocking event that occurred in the North Atlantic sector from 28 January to 9 February 1975 (Diao et al., 2006). It can be seen that large-scale westerly flow, which is strongly blocked

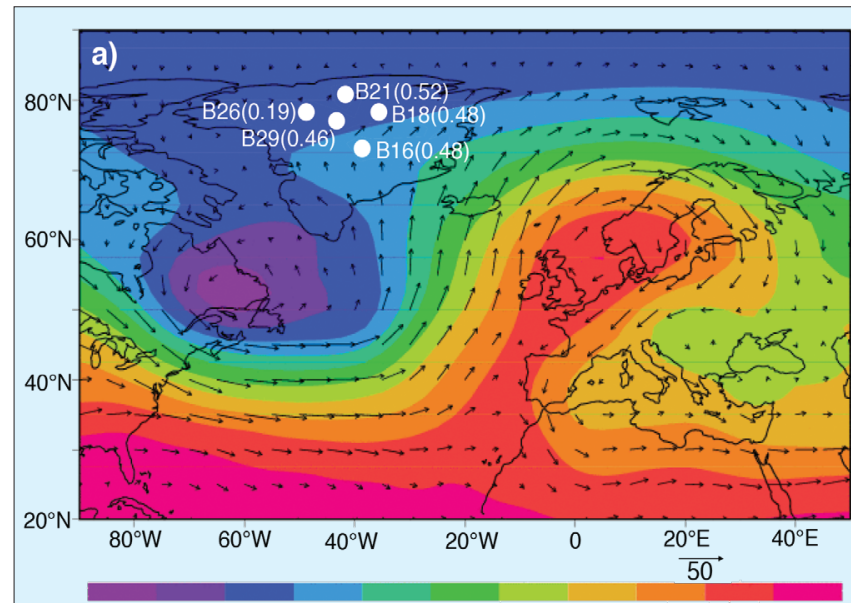

$\begin{array}{llllllllll}4900 & 5000 & 5100 & 5200 & 5300 & 5400 & 5500 & 5600 & 5700 & 5800\end{array}$

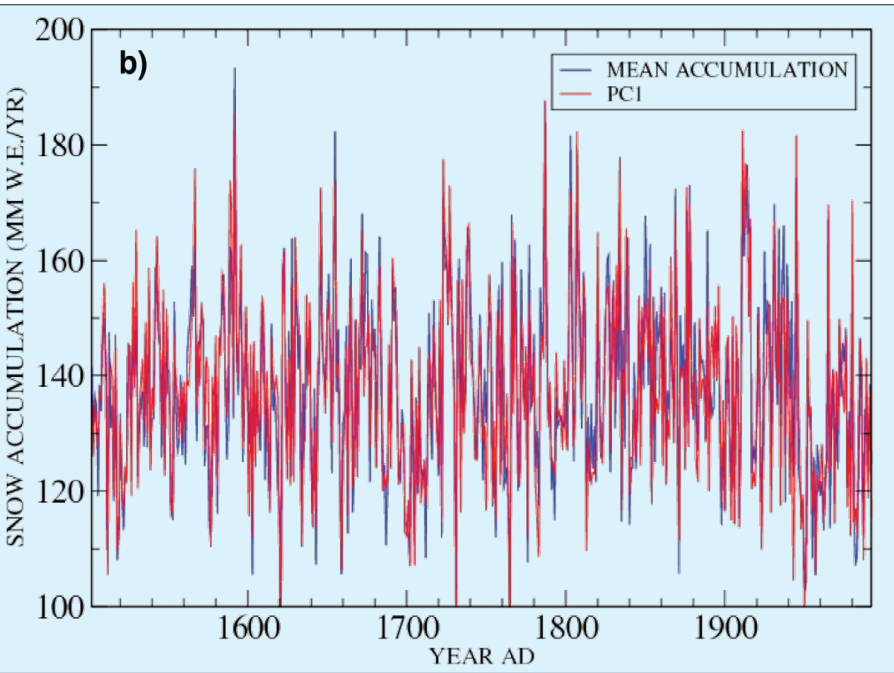

YEAR AD

Figure 1: a) The 500-hPa geopotential height (shaded; $m$ ) and horizontal wind (vector; $\mathrm{m} / \mathrm{s}$ ) during the mature stage (3 February 1975 ) of the $28 \mathrm{January}$ to 9 February 1975 blocking event that occurred in the North Atlantic region. Location of core sites (filled white circles) and EOF1 values of snow accumulation records from Greenland are also indicated. $\boldsymbol{b})$ The corresponding time coefficients (PC1) and the mean snow accumulation of the five records represented in a). The PC1 was scaled to have the same mean and variance as the mean accumulation time series ( $\mathrm{mm}$ w.e./yr $=\mathrm{mm}$ water equivalent per year). 